\title{
Quick Assessment of the Anomalies in Concrete Structure Using Dispersive Characteristic of Surface wave
}

\author{
Chia-Chi Cheng ${ }^{l}$, Keng-Tsang Hsu ${ }^{l}$, Chih-Hung Chiang ${ }^{l}$, Fong-Jhang Ke ${ }^{l}$, and Hong- \\ Hua Wang ${ }^{1}$ \\ ${ }^{1}$ Department of Construction Engineering, Chaoyang University of Technology, Taichung, Taiwan
}

\begin{abstract}
A waveform generated by a small impact hammer and recorded by single transducer is used to assess different kinds of anomalies in concrete. Using Short-Time Fourier Transform and the reassignment technique, the group slowness spectrogram can be calculated from the waveform. The surface wave response in the diagram of velocity vs. wavelength corresponding to the reassigned spectrogram is used for defect evaluation. Experiments were conduct on a concrete plate specimen containing multiple delaminated cracks, and a honeycomb. The group velocity profiles for test lines passing through different kinds of defects were obtained and compared. It was found the surface wave velocity begins to decrease with the increase of wavelength when the wavelength is about two times of the depth of the defect.
\end{abstract}

\section{Introduction}

Plate-like reinforced concrete structures, such as bridge deck, shear wall, or reinforced concrete containing wall of nuclear power station are highly related to structural safety. The internal anomalies may include honeycombs and air voids and delaminated cracks caused by improper casting, corrosion of steel bars, or excessive earthquake load. Although speedy assessment is possible, these anomalies may not be easily identified by high-frequency ground penetration radar (GPR). The electromagnetic waves emitted by GPR can travel through air. The opening of the air gap within $1 / 4$ of the wavelength in the media cannot be distinguishable [1]. For example, for GPR with $2.4 \mathrm{GHz}$ antenna, the distinguishable gap space needs to reach about $100 \mathrm{~mm}$ for air gap and $30 \mathrm{~mm}$ for gap filled with water. The impact-echo test method (IE) applies transient waves by hitting a steel ball on surface producing stress wave with broad frequency range. As an air gap, less than about $0.1 \mathrm{~mm}$, can produce noticeable reflection of the stress wave from the boundary $[2,3]$. The method is more sensitive than GPR in air void detection. The disadvantage of IE is time consuming. SASW (Spectral Analysis of Surface Waves) and MASW (Multichannel Analysis of Surface Waves) methods have also been used for assessing the material properties and thickness of layered structures such as asphalt pavement or concrete plate $[4,5]$. The instrumental setups require two receivers for SASW and multiple channels of receivers for MASW placing on the surface. In practice, multiple tapping on the surface by small hammer at different locations may be needed to obtain a dispersive phase velocity profile of 
surface wave. Using MASW method, not only the velocity profile of surface wave but also the dispersive characters of multiple Lamb wave modes of layered structure can be retrieved [4]. However, the SASW method requires changing the receivers' interval to obtain the wave speed for larger range of wavelength and the MASW method requires multiple measurements or multiple receivers to obtain the accurate phase information. Recently, a device which is conveniently operated at field was invented which combines the impact-echo method (IE) with the ultrasonic surface waves (USV) to evaluate the depth of surface deterioration and defects within concrete. Because the interval between receivers is fixed and the energy of ultrasound is limited, the variation of phase velocity with depth can be evaluated up to $30 \mathrm{~cm}$ [6].

The MASW, SASW and USV methods are designed to measure the phase velocity. In present study, the dispersive characteristics of group velocity of surface waves were used for investigation. Tests are performed with one receiver positioned away from the impacting source. The spectrogram of the group velocity obtained by the receiver is calculated by Short-Time Fourier Transform. The resolution of the spectrogram is enhanced by the reassigned technique. $[7,8]$ The advantage of the technique is the capability on fast investigation of large area, as the method only requires performing single test to obtain multiple lamb wave modal dispersive characteristics.

In the study, experiments were conduct on a plate specimen with delaminated crack, small range crack and honeycomb embedded inside. The group velocity profile of plain concrete, concrete containing different depths of delamination crack, concrete containing honeycombs were obtained and compared.

\section{Theoretical Backgrounds}

In present study, the technique involving only one test with single impacting source and single receiver is proposed. The signal is analyzed by the Short Time Fourier Transfer (STFT) and reassigning technique to obtain the spectrogram of the group slowness. The dispersive characteristics of group slowness of the test signal can be obtained by two steps. First, STFT was performed on the test signal to construct the preliminary spectrogram. Secondly, the images of dispersion characteristics in the spectrogram are enhanced by Time-Frequency Reassignment technique. For STFT, the signal $(\mathrm{s}(\mathrm{t}))$ is separated into a series of overlapping sections. Each section is multiplied by a select window function $(\mathrm{h}(\mathrm{t}))$ and transferred into frequency domain by FFT. The STFT, denoted as $\operatorname{Sh}(\omega, \mathrm{t})$, is defined as Eq. (1).

$$
S_{h}(\omega, t)=\frac{1}{2 \pi} \int_{-\infty}^{\infty} e^{-i \omega \tau} s(\tau) h(\tau-t) d \tau
$$

The spectrogram of $\operatorname{Sh}(\omega, t)$ is the energy density spectrum of a STFT, which can be calculated by Eq. (2).

$$
E_{d}(\omega, t)=|S(\omega, t)|^{2}
$$

In the reassignment method, high-energy corresponding to the dispersive character of a plate can be moved from its original location $(\omega, \mathrm{t})$ to a newly reassigned coordinates $(\hat{\omega}, \hat{t})$, which is usually at the center of gravity of the high energy band. The reassigned coordinates $\hat{\omega}$ and $\hat{t}$ for a spectrogram is shown in Eqs. (3) and (4) [7]. 


$$
\begin{gathered}
\hat{t}=t-\operatorname{Re}\left(\frac{S_{T h}(x, t, \omega) \cdot \overline{S_{h}(x, t, \omega)}}{\left|S_{h}(x, t, \omega)\right|^{2}}\right) \\
\hat{\omega}=\omega+\operatorname{Im}\left(\frac{S_{D h}(x, t, \omega) \cdot \overline{S_{h}(x, t, \omega)}}{\left|S_{h}(x, t, \omega)\right|^{2}}\right)
\end{gathered}
$$

Where Sh, SDh, STh are the STFT of the signal $\mathrm{x}$ using a normalized window function $\mathrm{h}(\mathrm{t})$, $\frac{d h(t)}{d t}$ and $\mathrm{t}^{*} \mathrm{~h}(\mathrm{t})$. The coding for reassigned technique can be found in [9].

\section{Experimental designs}

A specimen with dimensions $2 \mathrm{~m} \times 4.05 \mathrm{~m} \times 0.36 \mathrm{~m}$ was constructed with some artificial defects buried inside the normal weight concrete. As shown in Fig. 1, artificial cracks made by Styrofoam sheets were placed within concrete at different depths and size, so was a honeycomb block made by mortar bonded coarse aggregates. The honeycomb was wrapped by plastic sheet to prevent cement entering the voids during casting. The layout of defects is shown in Fig. 2. The figure also indicates the positions of the four test lines in present study. The four scenarios under the test lines are summarized by Table 1 .

The experiment was conducted with a special impact-hammer applying on one end of the test line. The produced stress waves were recorded by the displacement receiver at the other end of the test line. The distance between the impact-source and the receiver is $0.8 \mathrm{~m}$. In order to find the time origin of the impact, as shown in Figure 3(a), the impact-source is made by a steel half sphere embedded with piezoelectric element. The diameter of the half sphere applied in this study is $5 \mathrm{~mm}$ in diameter. For each test, a total of 4096 points was recorded in every $2 \mu \mathrm{s}$. The test setup is shown in Figure 3(b).

Table 1. The scenarios under the test line

\begin{tabular}{|c|c|c|}
\hline Test line Number & Situation beneath test line & Depth of the defect(s) \\
\hline 1 & No defect & Thickness $=0.36 \mathrm{~m}$ \\
\hline 2 & $\begin{array}{c}\text { horizontal Styrofoam disk with } \\
\text { diameter of } 0.5 \mathrm{~m}\end{array}$ & $0.19 \mathrm{~m}$ \\
\hline 3 & $\begin{array}{c}\text { horizontal Styrofoam disks with } \\
\text { diameters } 0.1 \mathrm{~m} \text { and } 0.2 \mathrm{~m}\end{array}$ & $\begin{array}{c}0.238 \mathrm{~m} \text { for } 0.2 \mathrm{~m} \text { dia. defect } \\
0.146 \mathrm{~m} \text { for } 0.1 \mathrm{~m} \text { dia. defect }\end{array}$ \\
\hline 4 & Honeycomb block & $0.127 \mathrm{~m}$ \\
\hline
\end{tabular}




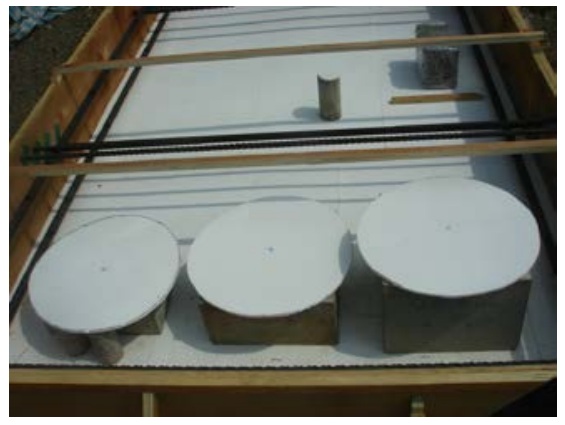

Fig. 1. Photo of defects buried in the specimen.

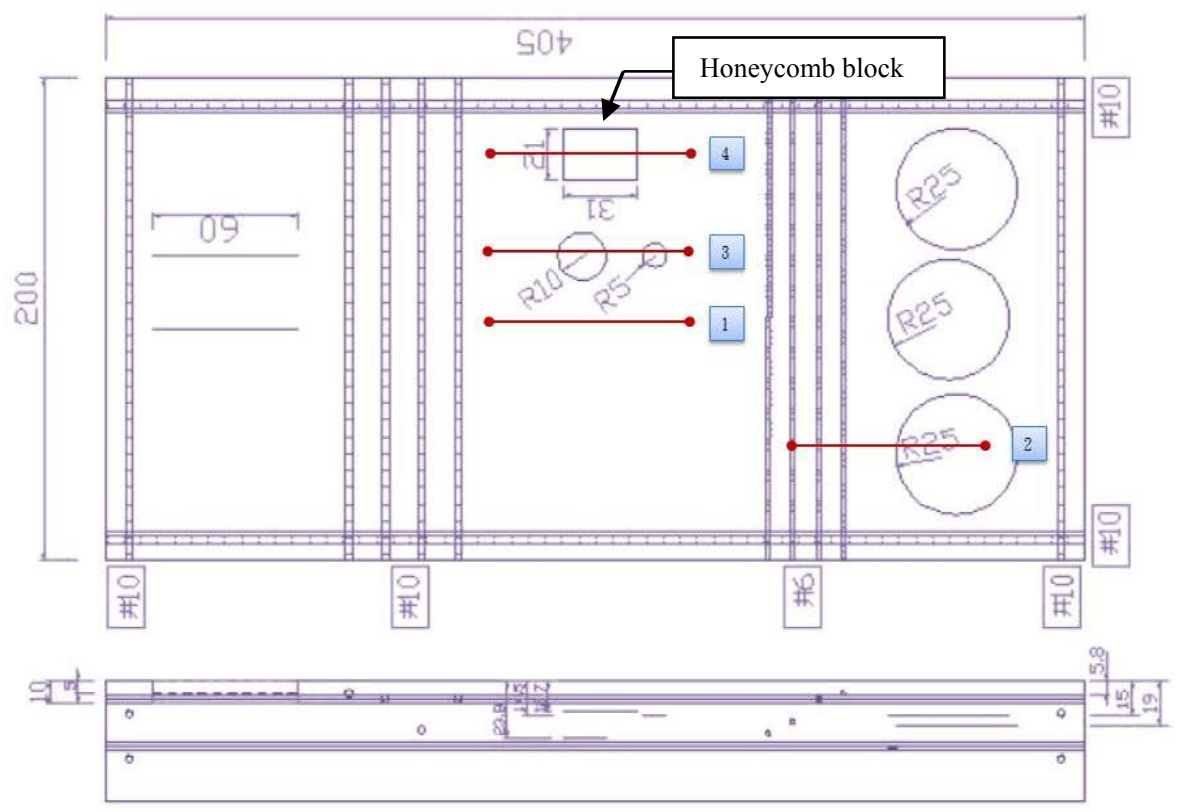

Fig. 2. Illustration of the specimen layout and positions of test lines.

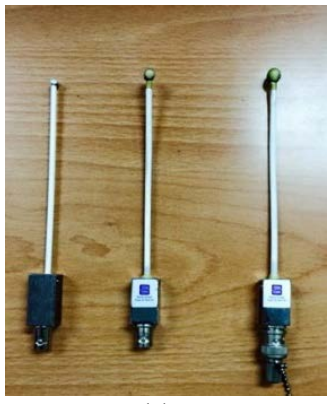

(a)

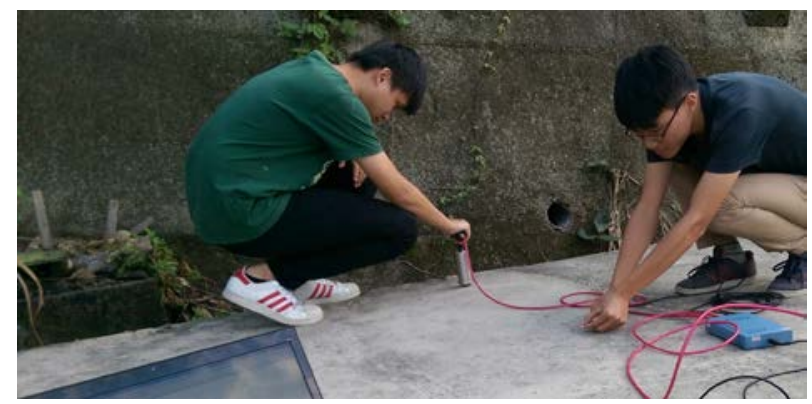

(b)

Fig. 3. Photos of (a) impactor embedded with piezo-electric element, (b) experimental setup.

\section{Experimental Results}

The algorithms to perform the analysis are as following. First, perform the STFT by chosen a Hanning window with certain window length and window overlap to obtain a 
spectrogram. Use the reassigned technique to find the center of gravity of the high energy band in the spectrogram. Third, divide the vertical time axis of the spectrogram to the impactor-receiver distance to obtain slowness axis. Fourth, obtain the velocity-wavelength diagram from the slowness spectrogram. Finally, the dominant response with the lowest slowness with respect to frequency in the slowness spectrogram would be shown as the dominant velocity profile with the largest velocity with respect to wavelength. The velocity profile is used for defect assessment.

The results for test lines 1 to 4 are shown in Figure 4 to 7 , respectively. Using Fig. 1 as the example, the right figure of Fig. 4(a) shows the waveform obtained from the displacement transducer $0.8 \mathrm{~m}$ from the impact hammer. The vertical axis of the waveform is the slowness, which is time divided by source-receiver distance. The spectrogram on the right of Fig. 4(a) is obtained by STFT and reassigned method. In STFT, the window size is 150 points and overlap of the window is 144 points. After reassigned the coordinates of the high energy band to the center of gravity in the spectrogram produced by STFT, the image of the response above $-40 \mathrm{~dB}$ of the maximum amplitude is calculated and shown in Fig. 4(a). Because the waveform and the spectrogram have the same vertical axis, the frequency contents of the major response in the waveform can be easily identified in the spectrogram. The darker dots means stronger signal. The diagram of velocity vs. wavelength obtained from the data in reassigned spectrogram is shown in Fig. 4(b).

The earliest major response corresponding to the arrival of the Rayleigh wave has noticeable difference for different defect-scenarios. In the reassigned spectrogram, the Rayleigh wave responses are around $0.4 \mathrm{~ms} / \mathrm{m}$ for frequency range $20-60 \mathrm{kHz}$ for all the test lines. The divergence caused by stress waves traveling around the defects occurs at the frequency range less than $20 \mathrm{kHz}$. For comparison, the theoretic solution of fundamental asymmetric Lamb wave mode (A0), fundamental symmetric Lamb wave mode (S0), and the second symmetric Lamb wave mode (S1) for a concrete plate with thickness $0.36 \mathrm{~m}, \mathrm{P}-$ wave speed of $4000 \mathrm{~m} / \mathrm{s}$, and poison ratio of 0.2 , are also shown in the spectrograms by red, orange, and blue lines.

For the case with no-defect, the Rayleigh wave response shown in Fig. 4(a) generally matches with the theoretical A0 mode at the frequency range larger than about $7 \mathrm{kHz}$. Below $7 \mathrm{kHz}$ the slowness graduate increases but the slowness remains around $0.4 \mathrm{~m} / \mathrm{s}$. For test line 2, which passes through a large delaminated disk at a depth of $0.19 \mathrm{~m}$, the Rayleigh wave response shown in Fig. 5(a) generally matches with the theoretical A0 mode at the frequency range $10-30 \mathrm{kHz}$ and a large increase in slowness can be found below $8 \mathrm{kHz}$. The slowness is little bit higher than the theoretical solution for frequency higher than $30 \mathrm{kHz}$. The test line 3 passes through two smaller horizontal Styrofoam disks with diameters $0.1 \mathrm{~m}$ and $0.2 \mathrm{~m}$ at the depths $0.238 \mathrm{~m}$ and $0.146 \mathrm{~m}$, respectively. As shown in Fig. 6(a), the Rayleigh wave response generally matches with the theoretical A0 mode at the frequency range $10-38 \mathrm{kHz}$ and a large increase in slowness can be found below $10 \mathrm{kHz}$. The slowness is a little bit higher than the theoretical solution for frequency higher than $38 \mathrm{kHz}$. For test line 4, which passes through a large honeycomb block at a depth of $0.127 \mathrm{~m}$, the Rayleigh wave response shown in Fig. 7(a) generally matches with the theoretical A0 mode at the frequency range $15-20 \mathrm{kHz}$. For frequency less than $10 \mathrm{kHz}$ the surface wave response seized to exist and a strong response can be found at the slowness of $0.6 \mathrm{~ms} / \mathrm{m}$.

The existence and the depth of the defect can be better recognized in the diagrams of velocity vs. wavelength. The velocity profiles of Rayleigh wave can be found as the dominant responses between $2000 \mathrm{~m} / \mathrm{s}$ and $3000 \mathrm{~m} / \mathrm{s}$. For the no-defect case shown in Fig. 4(b), the velocity is around $2500 \mathrm{~m} / \mathrm{s}$ for wavelength less than $0.2 \mathrm{~m}$, increases to about $2600 \mathrm{~m} / \mathrm{s}$ for wavelength between $0.2 \mathrm{~m}$ and $0.3 \mathrm{~m}$, and remains steady at $2600 \mathrm{~m} / \mathrm{s}$ for wavelength between $0.3 \mathrm{~m}$ and $0.4 \mathrm{~m}$. The velocity is gradually decreased to $2400 \mathrm{~m} / \mathrm{s}$ for wavelength between $0.4 \mathrm{~m}$ and $0.8 \mathrm{~m}$. For test line 2 shown in Fig. 5(b) with delaminated 
crack at the depth of $0.19 \mathrm{~m}$, again the velocity is around $2500 \mathrm{~m} / \mathrm{s}$ for wavelength less than $0.2 \mathrm{~m}$ and increases to about $2700 \mathrm{~m} / \mathrm{s}$ for wavelength between $0.2 \mathrm{~m}$ and $0.3 \mathrm{~m}$, and the begins to decrease at the wavelength of about $0.38 \mathrm{~m}$. The velocity decreases to $2200 \mathrm{~m} / \mathrm{s}$ for wavelength equal to $0.8 \mathrm{~m}$. For test line 3 shown in Fig. 6(b) with two cracks at the depths of $0.238 \mathrm{~m}$ and $0.146 \mathrm{~m}$, the velocity is around $2500 \mathrm{~m} / \mathrm{s}$ for wavelength less than $0.2 \mathrm{~m}$ and increases to about $2700 \mathrm{~m} / \mathrm{s}$ for wavelength between $0.2 \mathrm{~m}$ and $0.3 \mathrm{~m}$. The velocity begins to decrease at the wavelength of $0.31 \mathrm{~m}$ and to the value of $2000 \mathrm{~m} / \mathrm{s} \mathrm{for}$ wavelength reaching $0.5 \mathrm{~m}$. The velocity of the major response decreased to $1500 \mathrm{~m} / \mathrm{s}$ for wavelength between $0.5 \mathrm{~m}$ and $0.8 \mathrm{~m}$ as shown by green arrow in Fig. 6(b). For test line 4 shown in Fig. 7(b) with honeycomb block at the depth of $0.127 \mathrm{~m}$, the velocity is around $2500 \mathrm{~m} / \mathrm{s}$ for wavelength less than $0.18 \mathrm{~m}$. The surface wave velocity is around $2200 \mathrm{~m} / \mathrm{s}$ and $2500 \mathrm{~m} / \mathrm{s}$ for wavelengths between $0.18 \mathrm{~m}$ and $0.28 \mathrm{~m}$. When the wavelength reaches $0.28 \mathrm{~m}$, the major response near $2200 \mathrm{~m} / \mathrm{s}$ stopped and reappears at the velocity of $1700 \mathrm{~m} / \mathrm{s}$ as shown by the green arrow.

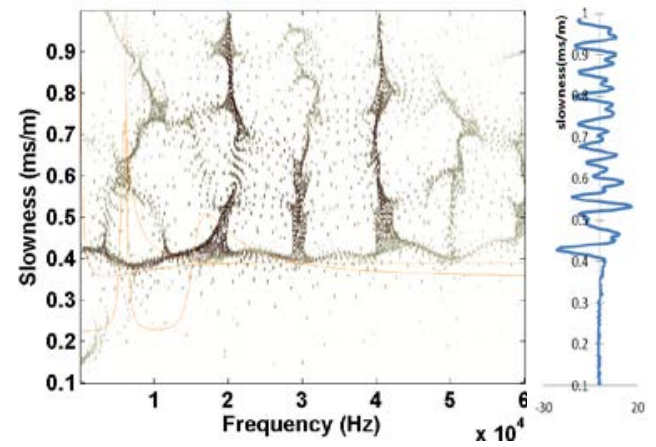

(a)

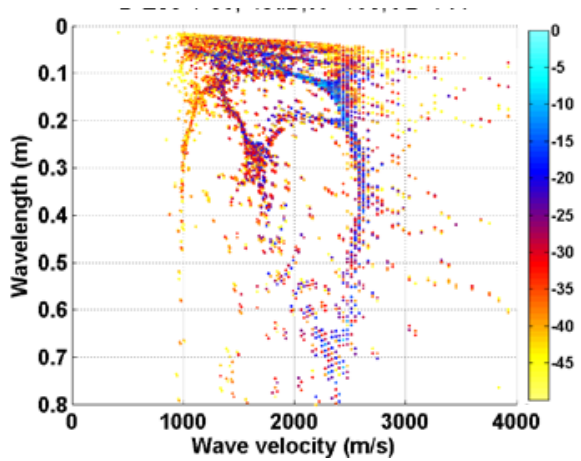

(b)

Fig. 4. Test line 1 (a) group slowness spectrogram along with waveform, (b) velocity-wavelength profile.

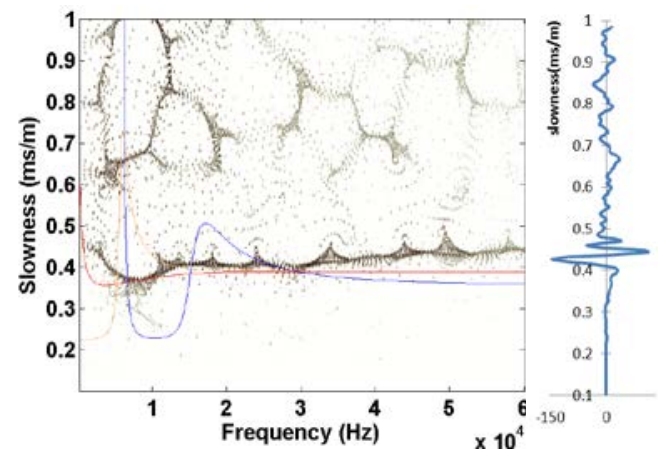

(a)

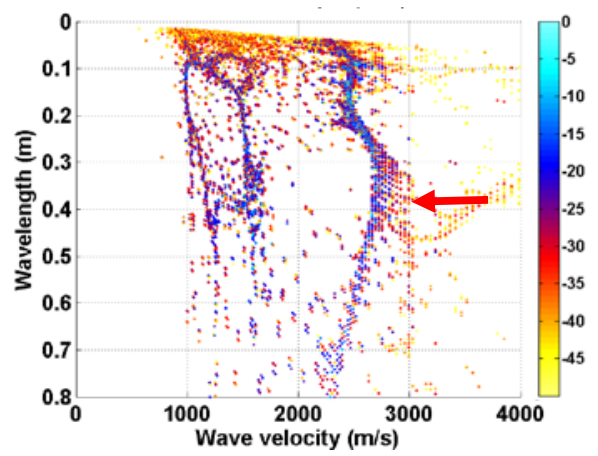

(b)

Fig. 5. Test line 2 (a) group slowness spectrogram along with waveform, (b) velocity-wavelength profile. 


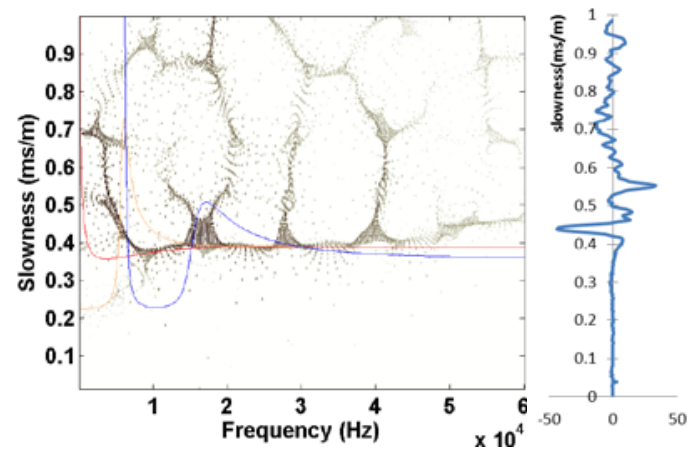

(a)

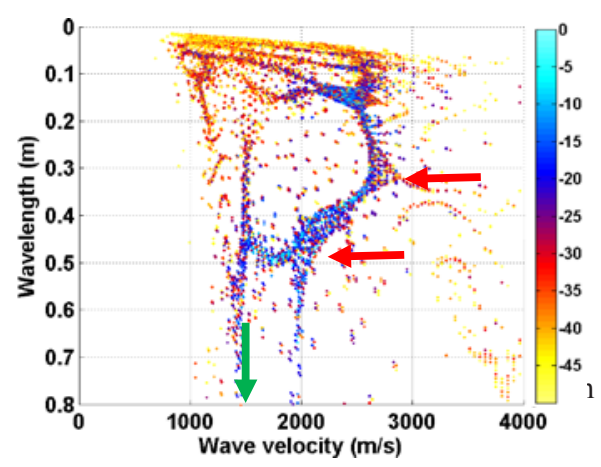

(b)

Fig. 6. Test line 3 (a) group slowness spectrogram along with waveform, (b) velocity-wavelength profile.

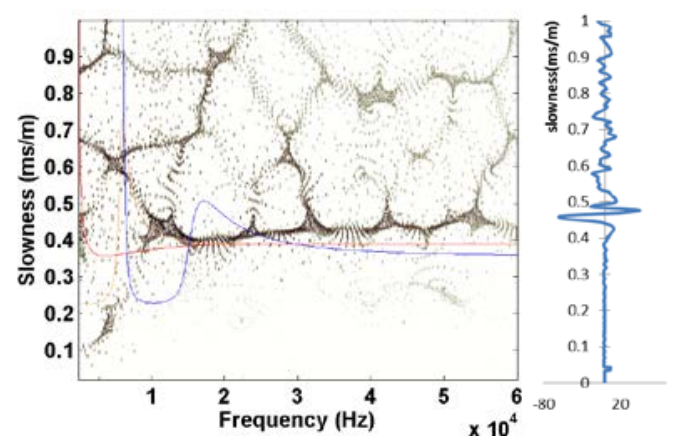

(a)

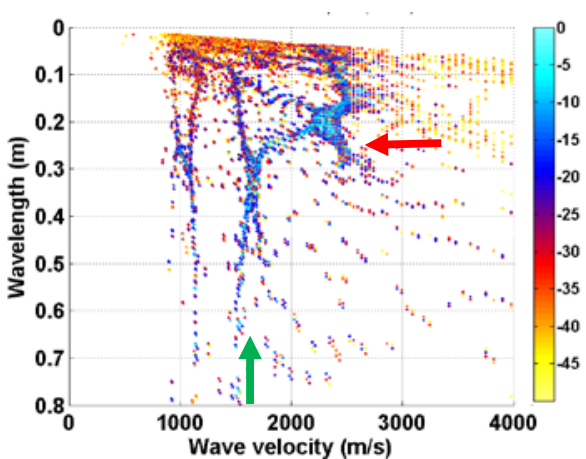

(b)

Fig. 7. Test line 4 (a) group slowness spectrogram along with waveform, (b) velocity-wavelength profile.

\section{Conclusions}

An algorithm to assess defects within a concrete plate can be developed from the surface wave velocity profiles obtained from present study. If the group velocity remains near 2400 $\mathrm{m} / \mathrm{s}$, there should be no defect under the test line. If the group velocity is under $2200 \mathrm{~m} / \mathrm{s}$ at the wavelength twice the depth of the plate, $0.72 \mathrm{~m}$ in present case, it may imply presence of defects under the test line. The group velocity of the surface wave gradually decreases for delaminated crack, and promptly decreases for the existence of honeycombs. As shown in Table 2, the velocity begins to decrease at the wavelength about 2 times of the depth of the defect for all the delaminated cracks and honeycomb. Thus, the depth of the defect under the test line can be estimated from the group velocity profile obtained from one recorded waveform. The impact source-receiver distance of $0.8 \mathrm{~m}$ is adequate for 
investigating defects contained in the $0.36 \mathrm{~m}$-thick concrete plate. As only one test using one receiver is required to investigate the anomalies under a test line of $0.8 \mathrm{~m}$, a quick and cheap assessment of concrete plate-like structure is made possible.

Table 2. Summarize the relationship between defect depth and the wavelength corresponding to decrease of wave velocity.

\begin{tabular}{|c|c|c|c|}
\hline Test line no. & (1) Depth of defect(s) & $\begin{array}{c}\text { (2) Velocity begins to } \\
\text { decrease at wavelength }\end{array}$ & $(2) /(1)$ \\
\hline 2 & $0.19 \mathrm{~m}$ & $0.38 \mathrm{~m}$ & 2 \\
\hline 3 & $0.146 \mathrm{~m}, 0.238 \mathrm{~m}$ & $0.31 \mathrm{~m}, 0.5 \mathrm{~m}$ & $2.1,2.2$ \\
\hline 4 & $0.127 \mathrm{~m}$ & $0.28 \mathrm{~m}$ & 2.2 \\
\hline
\end{tabular}

The authors would like to thank Ministry of Science and Technology, the Republic of China, Taiwan, for financially supporting this research under contract MOST 104-2221-E-324 -022 -MY3.

\section{References}

1. Meckel and Nath, Seismic Stratigraphy-applications to hydrocarbon exploration, (C. E. Payton, 1977), American Association of Petroleum Geologists, Tulsa.

2. C. Cheng, M. Sansalone, MATER STRUCT, 28 (1995), pp.74-82.

3. C. Cheng, M. Sansalone, MATER STRUCT, 28 (1995), pp.125-132.

4. R.A. Wardany, G. Ballivy, J-L Gallias, K. Saleh, J. Rhazi, ACI MATER J, 104, 3, (2007).

5. N. Ryden, M. Lowe, J ACOUST SOC AM, 116, 5 (2004).

6. H. Azari, S. Nazarian, ACI MATER J, 112, 4 (2015).

7. M. Niethammer, L. J. Jacob, J. Qu, J. Jarzynski, J ACOUST SOC AM, 107, 5, Pt.1 (2002).

8. S. Fulop, K. Fitz, J ACOUST SOC AM, 119, 1 (2006)

9. Kelly Fitz, Time-Frequency Analysis, 2008. Site includes code distributed under GNU public license. 